the thrush in our patient. Liver function tests during convalescence showed no evidence of liver damage.

In the early stage of the illness facilities were not available for conducting haematological or biochemical studies safely, so efforts were concentrated on establishing the virological diagnosis; in the late stage of the illness, when provision had been made for routine tests, ${ }^{16}$ they were not required for the management of the patient, though they proved useful for assessing the extent of damage during convalescence. Fortunately there was no bleeding and the use of prophylactic heparin was not considered to be necessary.

Once the haemoglobin and white blood cell levels had returned to normal plasmapheresis was performed to obtain a supply of convalescent serum.

We thank Professor $\mathrm{K}$ Cantell for supplying the interferon and Professor A J Zuckerman for advising on its use; the World Health Organisation team in the Southern Sudan and the International Commission team in Zaire for supplying the convalescent sera used in treatment ; Dr D A Rutter at the Microbiological Research Establishment, Porton, for the haematological and blood chemistry studies; and Dr Patricia A Webb at the Center for Disease Control, Atlanta, for some serological studies. Finally we would like to express our apprecia- tion of the support given by the staff of The Royal Free Hospital and the various health departments.

\section{References}

1 Martini, G A, Postgraduate Medical fournal, 1973, 49, 542

${ }^{2}$ Gear, J S S, et al, British Medical fournal, 1975, 4, 489.

3 Weekly Epidemiological Record, 1976, 51, 325.

4 Simpson, D, personal communication, 1977.

5 Johnson, K M, et al, Lancet, 1977, 1, 569.

6 Bowen, E T W, et al, Lancet, 1977, 1, 571.

' Pattyn, S, et al, Lancet, 1977, 1, 573.

${ }^{8}$ Emond, R T D, Postgraduate Medical fournal, 1976, 52, 563.

${ }^{9}$ British Medical fournal, 1976, 1, 64.

10 Bowen, E T W, British fournal of Experimental Pathology, 1969, 50, 400

${ }^{11}$ Fleming, W A, McNeill, T A, and Killin, T, Immunology, 1973, 23, 429.

12 Nissen, C, et al, Lancet, 1977, 1, 203.

13 McNeill, T A, and Gresser, I, Nature, New Biology, 1973, 244 (II), 173.

14 Falcoff, E, et al, fournal of Virology, 1973, 12, 421.

15 Johnson, H M, Smith, B G, and Baron, S, fournal of Immunology, 1975, 114, 403.

${ }^{16}$ Rutter, D A, British Medical fournal, 1977, 2, 24.

\title{
Prolonged remission maintenance in acute myeloid leukaemia
}

\author{
A S D SPIERS, J M GOLDMAN, D CATOVSKY, CHRISTINE COSTELlO, D A G GALTON, \\ C S PITCHER
}

British Medical fournal, 1977, 2, 544-547

TRAP programme must still be regarded as only palliative treatment for acute myeloid leukaemia.

\section{Summary}

Twenty-five patients with acute myeloid leukaemia were treated with three quadruple drug combinations in predetermined rotation: TRAP (thioguanine, daunorubicin, cytarabine, prednisolone); COAP (cyclophosphamide, vincristine, cytarabine, prednisolone); and POMP (prednisolone, vincristine, methotrexate, mercaptopurine). Fifteen patients $(60 \%)$ achieved complete remission and five $(20 \%)$ partial remission. For maintenance, five-day courses of drugs were administered every 14 to 21 days and doses were increased to tolerance. The median length of complete remission was 66 weeks. In eight patients remission maintenance treatment was discontinued and some remained in complete remission for over two years.

In this series the remission induction rate was comparable with that reported for other regimens and complete remission lasted longer with this intensive maintenance regimen than with others. Nevertheless, the

Medical Research Council Leukaemia Unit, Royal Postgraduate Medical School, London W12 0HS

A S D SPIERS, MD, FRACP, consultant physician (now professor, Section of Medical Oncology, University Hospital, Boston University Medical Centre, Boston)

J M GOLDMAN, BM, MRCP, consultant physician

D CATOVSKY, MD, MRCPATH, consultant physician

D A G GALTON, MD, FRCP, honorary director

CHRISTINE COSTELLO, MB, MRCP, research fellow

Stoke Mandeville Hospital, Aylesbury, Buckinghamshire HP21 8AL C S PITCHER, DM, FRCP, consultant haematologist

\section{Introduction}

Many regimens are used in the treatment of acute myeloid leukaemia (AML), but none has shown unique superiority. ${ }^{1-3}$ Intensive ${ }^{4}$ treatments have not proved greatly superior to nonintensive $^{5}$ regimens. Complex remission maintenance using multiple drugs ${ }^{6}$ may be little better than simpler ${ }^{7}$ regimens. Remission-induction programmes have incorporated single drugs $^{8}$ and combinations of seven ${ }^{9}$ or eight ${ }^{10}$ antileukaemic agents administered simultaneously. The complete remission rate in adults with AML has varied from $9.5 \%$ to $79 \%$ in different series. ${ }^{311}$ Higher complete remission rates have been reported for small groups of patients at specialised centres ${ }^{1112}$ than for larger groups treated at many hospitals, where rates have varied from $9.5 \%$ of 200 adults $^{3}$ to $34 \%$ of 301 adults. ${ }^{13}$ The importance of the choice of drugs and the intensity of treatment are outweighed by uncontrolled factors including patient selection and the differing capabilities of different institutions to give supportive care during the induction of remission. The complete remission rate in adults with AML has seldom exceeded $50 \%$ in a multicentre study and $65 \%$ in a specialised centre.

Attainment of complete remission in AML slightly improves survival. In large series the median duration of complete remission has varied from five to 11 months $^{313-15}$; median survival has been longer but has seldom exceeded 13 months. ${ }^{3}$ Immunotherapy administered during remission of $A M L^{16} 17$ seems to prolong the short duration of survival after relapse but does not prolong the duration of complete remission. No regimen for remission maintenance in $A M L$ is definitely superior, and the advisability of attempting to maintain remission at all has been questioned. 
We described ${ }^{18}$ a regimen for inducing and maintaining complete remission in AML, in which we applied principles derived from studies in animals and from clinical trials in children with acute lymphoblastic leukaemia. These principles were $(a)$ that drugs administered in combination were more effective than single agents for inducing remission ${ }^{19} ;(b)$ that the use of several drugs for remission maintenance could prevent or delay the emergence of leukaemia cells resistant to treatment ${ }^{20}$; and (c) that augmented doses of drugs were more effective for maintaining remission than lower doses. ${ }^{21}$ Remission was induced with a four-drug combination and maintained with three combinations, each of four drugs, in cyclical rotation. Patients with AML in complete remission with few leukaemia cells in the bone marrow tolerate chemotherapy better than patients in relapse. Accordingly the protocol provided for stepwise increases in the doses of antileukaemic drugs during remission. While the complete remission rate might not exceed that obtainable with other regimens, we hoped that the unusually intensive maintenance treatment, using all the first-line antileukaemic drugs at maximum permissible doses, might prolong the duration of complete remission. We report the results of this treatment programme in 25 patients followed for up to four years.

\section{Patients and methods}

Twenty-five patients ( 15 male, 10 female) entered the study between July 1970 and January 1976: we report the results at 31 January 1977. The patients' ages ranged from 2 to 63 years; 3 were aged under 20 and four were aged over 60 years old. The diagnosis of AML was based on the appearances of May-Grünwald-Giemsa-stained films of peripheral blood and bone marrow, supplemented by cytochemical staining of bone marrow cells. The cytological types of AML represented were myeloblastic (16), myelomonocytic (8), and monocytic (1). One patient had been treated for AML 24 months earlier but had had no treatment for 14 months before her relapse and subsequent inclusion in the present study. One patient had failed to respond to induction treatment with thioguanine and cytarabine for two months. The remaining 23 patients had received no treatment. One patient had AML secondary to a myeloproliferative disorder and one had acute on chronic myelomonocytic leukaemia.

Criteria for remission and length of remission-Complete remission and partial remission were defined as in the MRC trials, ${ }^{13}{ }^{22}$ and any improvement less than partial remission was classified as a therapeutic failure. The duration of remission was the time from the first bone marrow specimen showing complete remission to the first evidence of relapse, whether this was in marrow or peripheral blood.

Chemotherapy-Three combinations, each of four drugs, were used in rotation. Details of doses in each regimen are shown in table I. The TRAP regimen was derived from the drug cornbination tested in an MRC trial, ${ }^{13}$ but we substituted prednisolone for prednisone because the latter is a less active precursor of the former and thioguanine for mercaptopurine because of its more pronounced synergy with cytarabine. ${ }^{23}$ The COAP regimen was modified from the original combination ${ }^{24}$ in that the cyclophosphamide was given by mouth. The POMP regimen was also modified from the original schedule, ${ }^{10}$ in which all drugs were administered intravenously. By giving drugs by mouth when possible and administering cytarabine and methotrexate intramuscularly outpatient treatment was facilitated and patients could return to work. Each course of treatment necessitated one visit to the clinic, where vincristine or daunorubicin and the first injection of cytarabine or methotrexate were given. The remaining injections were given at home by the patient's own doctor, the visiting nurse, a family member, or the patient. This arrangement proved acceptable to patients and was also safe.

Doses and frequency of treatment-During complete remission we intended to increase the doses of drugs other than vincristine and prednisolone to maximum tolerable levels (table I). But doses were not increased or were slightly reduced if $(a)$ haemoglobin was consistently below $10 \mathrm{~g} / \mathrm{dl}$; (b) platelet counts were consistently below $75 \times 10^{\%} / 1$; or $(c)$ neutrophil counts were below $1 \times 10^{\circ} / 1$. Initially treatment was given every two weeks - that is, five days of treatment alternated with nine days without drugs. In 13 of the patients, however, the rest period was extended to 16 days because of recurrent neutropenia or thrombocytopenia, but the drugs were administered at full dose. ${ }^{21}$

Remission induction-Six courses of TRAP were given in 12 weeks. When complete remission was achieved maintenance was begun. If
TABLE I-TRAP regimen; COAP regimen, modified from that of Whitecar ${ }^{24}$; and POMP regimen, modified from that of Frei and Freireich ${ }^{19}$

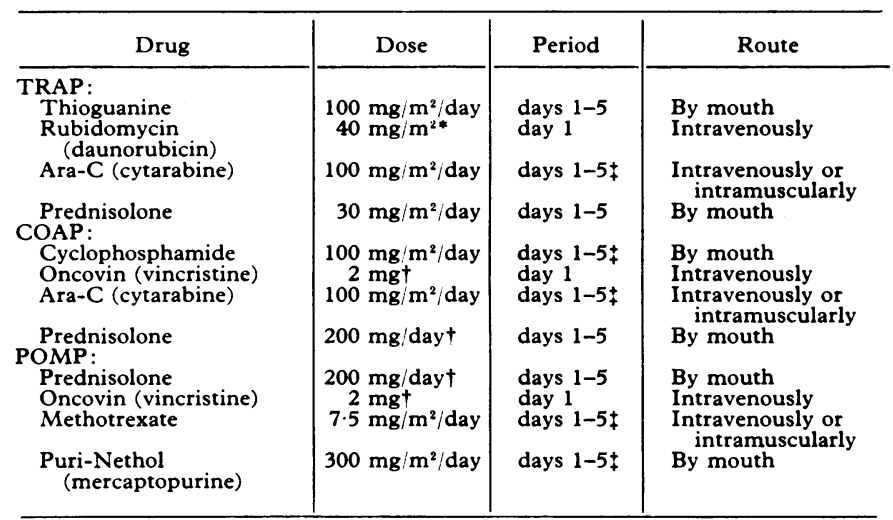

* If possible a $20^{\circ}$ o dose increase should be made on each course.

+There is no firm pharmacological basis for relating these doses to body surface area

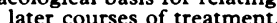

$\ddagger$ Usually given on days $1-3$ (first course), 1-4 (second course), and 1-5 (third and subsequent courses). Thereafter a $20 \%$ dose increase was attempted at each course. With TRAP the dose of thioguanine was increased in parallel; with COAP the doses of cyclophosphamide and cytarabine were increased in parallel; with POMP the doses of methotrexate and mercaptopurine were increased in parallel unless mucositis contraindicated increasing the dose of methotrexate.

partial remission had been reached further TRAP courses were given until complete remission was $\varepsilon$ chieved. If after six courses of TRAP there was not even partial remission the TRAP regimen was judged to have failed and COAP or other treatment was given.

Remission maintenance-Courses of treatment were administered as follows: two courses of COAP, followed by three courses of TRAP, followed by two courses of POMP, followed by three courses of TRAP. A complete cycle therefore included 10 courses of drug treatment and took 20-30 weeks: the cycle was then repeated. Because of the small number of investigators taking part breaches of the protocol by medical staff were negligible.

Monitoring-During remission induction the bone marrow was examined before each course of TRAP. During remission maintenance marrow was examined every four to six weeks.

\section{Results}

Induction of remission-Fifteen of the 25 patients $(60 \%)$ achieved complete remission and five $(20 \%)$ had partial remission. No particular age group or cytological subtype of AML was represented in the five patients in whom treatment failed. The median time from starting treatment to entering complete remission was 10 weeks (range 5-23 weeks), as in other studies. ${ }^{13}$

Length of complete remission-Complete remission in the 15 patients lasted from 28 to 208 weeks (mean 91 weeks; median 66 weeks). The numbers of patients remaining in complete remission at $6,12,18$, 24 , and 36 months were: $15(100 \%), 10(66 \%), 6(40 \%), 6(40 \%)$, and $2(13 \%)$ respectively. On 31 January 1977 two patients treated with the TRAP programme had been in complete remission for 119 and 134 weeks.

Survival-Patients who entered complete remission survived for 32 to 249 weeks (mean 123 weeks; median 102 weeks). On 31 January 1977 four patients were alive 100, 125, 200, and 249 weeks after diagnosis. Three were in remission; one of these had relapsed on the TRAP regimen and entered a further complete remission with even more intensive treatment. ${ }^{10}$

Discontinuing treatment-In eight patients maintenance treatment was discontinued during complete remission. The reasons for stopping treatment and the subsequent course are summarised in table II. In cases 1-3 treatment was discontinued for non-haematological reasons and complete remission continued for 42,124 , and 115 weeks. This prompted the trial in four patients of electively discontinuing treatment after one year of complete remission, ending with intensive consolidation treatment. This consisted of two successive seven-day intravenous infusions of cytarabine, each accompanied by three consecutive doses of daunorubicin. ${ }^{4}$ These four remissions lasted, without further treatment, for 14,14, 56, and 67 weeks, the last persisting on 31 January 1977.

Experience of relapse-Thirteen of the 15 patients in complete remission relapsed: six relapses occurred during maintenance treat- 
TABLE II-Details of eight patients with $A M L$ whose maintenance treatment was discontinued during complete remission

\begin{tabular}{|c|c|c|c|c|c|c|}
\hline \multirow{2}{*}{$\begin{array}{l}\text { Case } \\
\text { No }\end{array}$} & \multirow{2}{*}{\multicolumn{3}{|c|}{$\begin{array}{l}\text { Reason for discontinuing } \\
\text { remission maintenance }\end{array}$}} & \multicolumn{3}{|c|}{$\begin{array}{c}\text { Duration of complete remission } \\
\text { (weeks) }\end{array}$} \\
\hline & & & & $\underset{\text { On }}{\text { Oreatment }}$ & $\begin{array}{c}\text { Off } \\
\text { treatment }\end{array}$ & Total \\
\hline $\begin{array}{l}1 \\
2 \\
3 \\
4 \\
5 \\
6 \\
7 \\
8\end{array}$ & \multicolumn{3}{|c|}{$\begin{array}{l}\text { Refused maintenance treatment } \\
\text { Daunorubicin cardiotoxicity } \\
\text { Cerebrovascular accident } \\
\text { Maximum permissible daunorubicin } \\
\text { Sustained complete remission for } 1 \text { year* }\end{array}$} & $\begin{array}{r}1 \\
62 \\
93 \\
122 \\
52 \\
52 \\
52 \\
52\end{array}$ & $\begin{array}{c}42 \\
124 \\
115 \\
17 \\
14 \\
14 \\
56 \\
67 \dagger\end{array}$ & $\begin{array}{c}43 \ddagger \\
186 \\
208 \\
139 \\
66 \\
66 \\
108 \\
119+\end{array}$ \\
\hline
\end{tabular}

*These four patients received a final intensive consolidation treatment with daunorubicin and cytarabine immediately before discontinuing maintenance.

†Patient still in remission at 31 January 1977.

$\ddagger$ Patient still alive, in relapse, 92 weeks after stopping treatment.

ment and seven after treatment had been discontinued. Most of these patients received intensive multiple-agent chemotherapy, ${ }^{10}$ but only one patient in each group (maintenance and no-maintenance) entered a further complete remission.

Toxicity-Nausea and vomiting were almost universal with the TRAP regimen, less common with COAP, and uncommon with POMP. Paraesthesiae and constipation were uncommon after COAP and POMP, probably because only two doses of vincristine were given, two weeks apart, in 10 weeks. Although 15 patients were aged 40 to 63 years, the large doses of steroid did not provoke complications, presumably because steroid administration was brief and intermittent. Stomatitis accompanying the POMP regimen precluded the use of more than four daily doses of methotrexate in most patients. All regimens caused alopecia but hair regrew despite continuing treatment. One patient developed cardiac failure attributed to daunorubicin and improved over several months after discontinuing treatment. During remission induction bone marrow hypoplasia and pancytopenia occurred in all cases before complete remission was attained, as with all of the multiple-drug regimens we have used in AML. Pancytopenia during remission maintenance was uncommon and usually associated with intercurrent viral infection or with the impending relapse of AML. The dose of mercaptopurine in the original POMP protocol ${ }^{19}$ was poorly tolerated; by giving $40 \%$ less $\left(300 \mathrm{mg} / \mathrm{m}^{2} /\right.$ day for five days) we prevented protracted pancytopenia occurring after the second of each pair of POMP treatments. Evidence of hepatocellular damage sometimes occurred after POMP, perhaps because the large doses of mercaptopurine were administered orally instead of intravenously as in the original protocol. Despite the side effects only one patient refused maintenance treatment, and patients in complete remission returned to work and to near-normal domestic and social life.

\section{Discussion}

The TRAP regimen combines four drugs that are effective in AML and have different mechanisms of action. ${ }^{25-28}$ There is demonstrable synergy between cytarabine, thioguanine, and daunorubicin. ${ }^{23} 29$ Administration of drugs in five-day courses with intervals of nine to 16 days allows the effects of each course to be assessed before the next is begun and allows regeneration of normal bone marrow. The $60 \%$ complete remission rate in our patients was acceptable but not exceptional for a small series, ${ }^{11}{ }^{30}$ and a multicentre trial is necessary to confirm this in general hospital practice. In one such study with a similar regimen, where mercaptopurine was used instead of thioguanine, 37 out of 118 adults $\left(31 \%\right.$ ) with AML achieved complete remission. ${ }^{13}$ The TRAP regimen was used in the MRC's sixth trial in adult $A M L$, and preliminary results suggest that it produces better results than the combination of cytarabine and daunorubicin.

The remission maintenance phase of our programme was unusually intensive: we gave four-drug combinations in frequent courses of treatment and increased the doses of drugs to tolerance on the assumption that the bone marrow of patients in complete remission withstands more treatment than would be safe during relapse. During complete remission each patient received all the agents most useful in $A M L$, with the aim of preventing drug resistance. We hoped that these stratagems might continue the reduction in leukaemic-cell mass which had been started by the induction of complete remission, with a resulting prolongation of complete remission or even eradication of the leukaemic cells. Similar concepts underly other intensive multiple-drug maintenance schedules in the acute leukaemias. ${ }^{30} 31$

Complete remission in our patients lasted a median of 66 weeks (mean 91 weeks; range 28-208 weeks). This compares favourably with median durations of 26 weeks in MRC trials, ${ }^{13}$ 20 and 28 weeks in Acute Leukaemia Group B studies, ${ }^{29}$ and 42 weeks in patients treated with the L-6 protocol at Memorial Hospital. ${ }^{30}$ Of the above regimens only the L-6 is as intensive as the TRAP programme. Administering only hydroxyurea as maintenance treatment, the median duration of complete remission was 28 weeks. ' In a small series $79^{\circ}{ }_{\circ}$ of adults with AML achieved complete remission but the median duration of complete remission was only 40 weeks. ${ }^{11}$ Median durations of complete remission of 27 and 44 weeks were reported for patients who received, respectively, non-intensive chemotherapy and identical chemotherapy together with active immunotherapy. ${ }^{32}{ }^{33}$ The efficacy of the TRAP programme for maintaining complete remission in AML thus seems superior even when compared with other small series treated at specialised centres.

An Italian group treated adults with AML using a modified TRAP programme, omitting POMP from the maintenance phase. ${ }^{34}$ Thirteen out of 27 patients $\left(48^{\circ}{ }_{\mathrm{o}}\right)$ achieved complete remission. The median duration of complete remission had not been reached in their report. Five out of eight patients aged over 60 years entered complete remission, and the authors concluded that TRAP was more suitable than other treatments for elderly patients with AML.

A modest improvement in the length of complete remission and survival does not necessarily justify the inconvenience and morbidity of prolonged intensive chemotherapy, but this decision must be the patient's, and only one of our patients declined maintenance treatment. Only two of the 13 patients who relapsed achieved a further complete remission, indicating that after intensive treatment with all the major drugs effective in AML most patients are highly resistant. Nevertheless, the long initial remissions justify the use of multiple drugs for maintenance. Prolonged unmaintained complete remission in some patients after treatment was stopped (table II) suggests that the TRAP programme, with or without final consolidation treatment, reduces the body load of leukaemia cells to low levels in some cases, although the AML cells were not eradicated.

Even if these results are confirmed in larger series, the TRAP programme must still be regarded as only palliative treatment for AML. The cumulative dose of daunorubicin prohibits its very protracted use and a year of treatment does not destroy every AML cell. Substitution of doxorubicin for daunorubicin would reduce the risk of cardiotoxicity, and use of the regimen for inducing and consolidating complete remission before giving a final intensive consolidation treatment and then stopping treatment ${ }^{35}$ merits further study.

Requests for reprints should be addressed to Professor A S D Spiers, Section of Medical Oncology, University Hospital, Boston University Medical Centre, 75 East Newton Street, Boston, Massachusetts 02118 , USA.

\section{References}

1 Henderson, E S, Seminars in Hematology, 1969, 6, 271.

2 Spiers, A S D, in Leukemia, ed F Gunz and A G Baikie, p 655. New York, Grune and Stratton, 1974.

3 Enck, R E, Bauman, A W, and Bennett, J M, Archives of Internal Medicine, 1976, 136, 1256.

- Yates, J W, et al, Cancer Chemotherapy Reports, 1973, 57, 485.

5 Burge, P S, et al, Lancet, 1975, 2, 621.

- Clarkson, B D, Cancer, 1972, 30, 1572

7 Bloomfield, C D, et al, Cancer, 1973, 31, 931. 
${ }^{8}$ Bernard, J, Jacquillat, C, and Weil, M, Seminars in Hematology, 1972, 9,181 .

${ }^{9}$ Brincker, H, Scandinavian fournal of Haematology, 1975, 14, 35

10 Spiers, A S D, et al, Cancer, In press.

11 Gale, R P, and Cline, M J, Lancet, 1977, 1, 497.

12 Crowther, D, et al, British Medical fournal, 1970, 2, 513.

13 Medical Research Council's Working Party on Leukaemia in Adults, British fournal of Haematology, 1974, 27, 373.

14 Wiernik, P H, and Serpick, A A, Medicine, 1970, 49, 505.

16 Beard, M E H, and Fairley, G H, Seminars in Hematology, 1974, 11, 5.

16 Crowther, D, et al, British Medical fournal, 1973, 1, 131.

12 Gutterman, J U, et al, Lancet, 1974, 2, 1405.

18 Spiers, A S D, Clinics in Haematology, 1972, 1, 127.

19 Frei, E, and Freireich, E J, Advances in Chemotherapy, 1965, 2, 269.

${ }^{20}$ Pinkel, D, fournal of the American Medical Association, 1971, 216, 648.

21 Pinkel, D, et al, Cancer, 1971, 27, 247.

2.2 Medical Research Council's Working Party on Leukaemia in Adults, British Medical fournal, 1963, 1, 7.
${ }^{23}$ Carey, R W, Cancer Research, 1970, 11, 15.

${ }^{24}$ Whitecar, J P, Bodey, G P, and Freireich, E J, Cancer Research, 1970, 11, 83.

${ }^{25}$ Murphy, M L, et al, Cancer Research, 1955, 2, 36.

${ }^{26}$ Boiron, M, et al, Lancet, 1969, 1, 330.

27 Goodell, B, Leventhal, B, and Henderson, E, Clinical Pharmacology and Therapeutics, 1971, 12, 599.

${ }^{28}$ Medical Research Council's Working Party on Leukaemia in Adults, British Medical fournal, 1966, 1, 1383.

${ }^{29}$ Acute Leukemia Group B, Cancer, 1975, 36, 1560.

${ }^{30}$ Clarkson, B D, et al, Cancer, 1975, 36, 775.

${ }^{31}$ Spiers, A S D, et al, British Medical fournal, 1975, 4, 614.

${ }^{32}$ Fairley, G H, British fournal of Haematology, 1975, 31, suppl, p 181.

${ }^{33}$ Powles, R L, et al, British fournal of Cancer, 1977, 35, 265.

${ }_{34}$ Paolino, W, et al, British Medical fournal, 1973, 3, 567.

${ }^{35}$ Freireich, E J, et al, Archives of Internal Medicine, 1976, 136, 1417.

\title{
Contribution of atenolol, bendrofluazide, and hydrallazine to management of severe hypertension
}

\author{
R G WILCOX, J R A MITCHELL
}

British Medical fournal, 1977, 2, 547-550

\section{Summary}

The efficacy of various combinations of atenolol, bendrofluazide, and hydrallazine given twice daily was assessed in a double-blind trial on 29 patients with moderate to severe essential hypertension. Concurrent treatment with all three drugs proved most effective and produced a mean reduction in blood pressure of $43 / 31 \mathrm{~mm} \mathrm{Hg}$. In the dosage used, hydrallazine affected only the diastolic blood pressure, and when added to either bendrofluazide or bendrofluazide plus atenolol it produced a further mean reduction in pressure of $6 \mathrm{~mm} \mathrm{Hg}$. Once-daily treatment with atenolol and bendrofluazide was as effective in reducing blood pressure as the same combination given twice daily, and the hypotensive effect was still present at least 24 hours after the last dose of tablets. A combined tablet of atenolol and bendrofluazide taken once daily would be a simple regimen to follow and would provide almost as much hypotensive effect as a twice-daily regimen incorporating a modest dose of hydrallazine.

The hypotensive effect of atenolol was equal to that of bendrofluazide on systolic pressure but significantly better than that of bendrofluazide on diastolic pressure. Atenolol reduced plasma renin and urate concentrations but increased plasma potassium levels. The biochemical effects of atenolol, therefore, may be an advantage over those of bendrofluazide when deciding on first-line treatment for essential hypertension.

\section{Introduction}

Since hypertension is nearly always symptomless a complex treatment regimen that causes side effects is unlikely to be acceptable to patients. Because beta-blocking agents have a low

Department of Medicine, General Hospital, Nottingham NG1 6HA R G WILCOX, BSC, MRCP, lecturer in medicine

J R A MITCHELI, PHD, FRCP, professor of medicine

incidence of side effects they are widely used in hypertension. They may be combined with diuretics ${ }^{1}$ or with peripheral vasodilators $^{2}$ to achieve an enhanced hypotensive effect, but this increases the number of tablets to be taken each day. Atenolol is a cardioselective beta-blocker, and it is effective when given once daily to patients with mild to moderate hypertension.' We set out to evaluate the relative contribution of each component of a beta-blocker-diuretic-peripheral vasodilator combination to the management of patients with more severe degrees of high blood pressure and to determine whether the most effective components of such a combination could be given once a day. The effect of the various hypotensive combinations on plasma renin and other biochemical indices was also investigated.

\section{Patients and methods}

Twenty-nine patients ( 22 men, 7 women) with non-malignant essential hypertension took part in the study. Informed consent was obtained in each case and the study was approved by the hospital's ethical committee. Most of the patients were already on hypotensive treatment and were referred because of difficulty with control or problems with unwanted drug effects.

Figure 1 shows the design of the trial. At the first referral visit any current treatment was stopped, and the patients were then seen every two weeks for the next eight weeks. After the first four weeks they were given placebo tablets resembling atenolol, bendrofluazide, and hydrallazine to take twice daily. The bendrofluazide placebo contained riboflavin, which is excreted in the urine and causes it to fluoresce under ultraviolet light, and this was used as a simple test of compliance during the next four weeks. During this run-in period the acetylator status of each patient was assessed with the use of Schroder's4

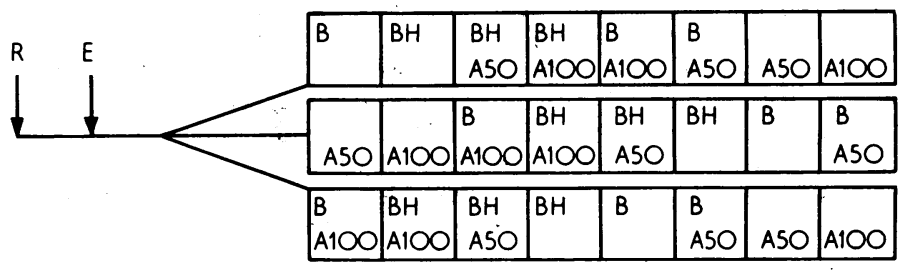

FIG. 1-Trial design. $R=$ First referral visit. $E=$ Entry to placebo period four weeks later. $\mathrm{B}=$ Bendrofluazide. $\mathrm{H}=$ Hydrallazine. $\mathrm{A} 50, \mathrm{~A} 100=$ Atenolol 50 and $100 \mathrm{mg}$. 